\title{
IX.
}

\section{Aus der Privatklinik des Dr. Rydygier in Kulm a.W.}

\section{Zur Sprayfrage.}

\author{
Von \\ Dr. Rydygier \\ in Kulm a.W.
}

In neuester Zeit haben sich kurz hintereinander drei Autoren mit der Sprayfrage beschäftigt: ein Zeichen, dass dieselbe im hohen Grade das Interesse der Fachgenossen auf sich gelenkt hat. Alle drei Stimmen haben sich gegen den Spray erklärt: Trendelenburg ${ }^{1}$ und von Bruns ${ }^{2}$ ) auf Grund ihrer praktischen Erfolge; Mikulicz ${ }^{3}$ ) auf theoretische Erörterungen und Experimente gestützt.

Es sei mir gestattet, im Nachfolgenden Einiges zur Vertheidigung des Spray beizutragen.

Dabei will ich vorläufig die beiden Arbeiten von Trendelenburg und von Bruns unberiteksichtigt lassen, da sich gegen dieselben nichts weiter sagen lässt, als was schon $\mathrm{Maas}^{4}$ ) in seinem Referate über die Trendelenburg'sche Arbeit angeführt hat, dass nämlich Trendelenburg - nnd ebenso auch von Bruns - dadurch nur zeigen, dass sie unter den Verhältnissen ihres Krankenhauses den Spray entbehren können, wie A. Bidder dies auch für die Verhältnisse seiner Privatpraxis angegeben hat. Trotzdem Trendelenburga) später die durchaus nicht giinstigen hygienischen Ver-

1) Ueber die Bedeutung des Spray für die antiseptische Wundbehandlung. Langenbeck's Archiv. 24. Bd. S. 779.

2) Fort mit dem Spray! Berlin. klin. Woclenschrift. 1880. Nr. 43.

3) Zur Sprayfrage. Langenbeck's Archiv. 25. Bd. S. 707.

4) Referat über die Trendelenburg'sche Arbeit im Centralblatt für Chir. 1880. Nr. 5.

5) Nachträgliche Notiz zu dem Aufsatz über die Bedeutung des Spray für die antiseptische Wundbehandlung. Langenbeck's Archir. 25. Bd. S. 483. 
hältnisse des Rostocker Krankenhauses darzulegen versucht, so gibt er uns gerade am Schluss jener Auseinandersetzung eine Waffe in die Hand, die wir später noch des Weiteren gegen die Gegner des Spray - wenigstens gegen die volle Gültigkeit des praktischen Beweises - ausnutzen werden, dass nämlich durch das stricte Durchführen der antiseptischen Wundbehandlung durch K önig, den Vorgänger Trendelenburg's, die inneren Verbältnisse des Rostocker Krankenhauses trotz der nicht besonderen äusseren Verbältnisse so brillant wurden ("die Verhältnisse werden mit einem Schlage besser"), dass Trendelenburg jetzt sehr selten in der Lage ist, den Studirenden ein Erysipel zeigen zu können. Diese verbesserten Verhältnisse bestanden zur Zeit, als Trendelenburg obne Spray anfing zu operiren; es ist sehr fraglich, ob Trendelenburg ebenso so gute Resultate, wie die publicirten, erzielt hätte, wenn er den Spray zu Zeiten, als die Verhältnisse der chirurgischen Klinik in Bezug auf Wundverlauf' und Wundkrankheiten recht mittelmässige waren und Erysipele noch häufig und in sehr schwerer Form auttraten, fortgelassen hätte.

Eben dasselbe kann man auch von v. Bruns sagen: auch er fing erst den Spray an fortzulassen, nachdem er mehrere Jahre vorher in seiner Klinik den ganzen Lister'schen Apparat nebst dem Spray angewendet hat; den letzteren freilich mehr um blos nunbegründete Vorwürfe wegen Unterlassung des so wichtigen Carbolspray von meiner Klinik abzuhalten ". Es wird mir ein Jeder zugeben, dass man in solchen Kliniken manche Unterlassungssiunde gegen die Antisepsis begehen kann, ohne dass sie sich sofort rächt - doch davon noch später.

Jetzt wollen wir uns zuerst auf das theoretische Raisonnement einlassen und vor Allem die Arbeit Mikulicz's im Auge behalten. Ohne des Näheren auf seine theoretischen Auseinandersetzungen über Wundinfection im Allgemeinen einzugehen, wollen wir hier gleich zu Anfang hervorheben, dass die ganze Beweisführung Mikulicz's dadurch eine mangelhafte ist, dass er von vornherein jede andere Einwirkung des Spray ausschliesst resp. unberiucksichtigt lässt, als die, welche der Spray auf die in der Luft suspendirten Infectionskeime hat. Um die Wichtigkeit des Spray herabzusetzen, führt er an, dass die Gefahr der Luftinfection gegenüber anderer Infection, wie der durch Hände, Pincetten, Instrumente u. s. w. eine minimale sei, und doch glaube ich, bestimmt behaupten zu können, dass gerade der Spray es ist, welcher in letzter Minute noch die Gefahr abzuwenden im Stande ist, wenn wir es verabsäumt haben, unsere 
Hände u. s. w. genau zu reinigen oder sie zufällig während der Operation verunreinigt haben: die feinen Spraytropfen vereinigen sich zum Regen, der entweder die anhaftenden Keime abspült oder deren Lebensfähigkeit herabsetzt. Jeder von uns weiss, dass von der Wunde, den Händen und Instrumenten die Sprayflüssigkeit in bedeutender Quantität abfliesst, so dass sie wobl im Stande ist, die feinen Infectionskeime abzuspiulen.

Eine Beweisführung gegen die Nütslichkeit des Spray, die blos die Luftinfection berïcksichtigt, wie die Mikulics'sche, ist also schon in ihrer Anlage mangelhaft.

Der Spray hat auf die "Contactinfection" entschieden hindernden Einfluss. Eine andere Frage ist die, ob man in dieser Beziehung nicht den Spray durch mehr oder minder hänfige Irrigation vertreten könnte, ferúer was für den Chirurgen von beiden lästiger und für den Kranken schädlicher sei. Doch davon später; gehen wir jetzt zur Frage ïber, welchen Einfluss der Spray auf die Luftinfection hat. Hierbei miissen wir eine doppelte Wirkungsweise desselben unterscheiden und im Auge behalten: 1) die mechanische und 2) die chemische. Die mechanische Wirkung besteht darin, dass der Sprayregen als solcher ohne Rlicksicht auf das Desinficiens in Form feinster Tropfen auf die Wunde und deren Umgebung niederfällt. Diese Wirkungsweise ist selbstverständlich gar nicht durch die Irrigation, die sonst als Ersatz vorgeschlagen wird, zu ersetzen. Von ihr behauptet aber Mikulicz, dass sie der Wunde nichts nützt, sondern im Gegentheil schadet. $\mathrm{Er}$ sagt darüber S. 735 wörtlich Folgendes:

"Es kann keinem Zweifel unterliegen, dass der besprochene mechanische Act des Spray, wenn er in Rechnung kommen soll, an und für sich der Wunde nichts nützen, sondern nur schaden kann, indem er die Luftkeime, die sonst nur spärlich zur Wunde gelangen, in grosser Masse niederschlägt." $\mathrm{Zu}$ diesem Schluss ist Mikulicz in Folge einer Reihe von Experimenten gekommen: Kohlenstaub wurde in einem abgegrenzten Raum durch Blasebälge "continuirlich" in die Luft gestäubt. Am anderen Ende wurden acht flache Gefässe aufgestellt in gleicher Entfernung von der Staubquelle. Eine bestimmte Koblenmenge wurde als Einheit angenommen und nach 5 Minuten betrug der auf die Gefässe niedergefallene Staub zwischen 5-10 Einheiten, im Mittel 81/2.

Darauf liess er den Zerstäubungskegel eines Handspray über die Reihe der Gefässe streichen und nun betrug die in 5 Minuten herabgefallene Staubmenge im ersten Gefäss 36 Einheiten, im zwei- 
ten 29, in den weiteren 25, 21, 17, 12, 11 und 7. "Der Staubniederschlag war also unter dem Einfluss des Sprayapparates im ersten Gefässe ungefähr vervierfacht worden und nahm dann allmählich an Quantität $a b$; in den letzten ausserhalb der Sphäre des Zerstäubungskegels liegenden Gefässen, blieb die Menge ungefähr so gross, wie in der vorangehenden Versuchsreihe ", d. h. als man die Menge ohne Einwirkung des Spray bestimmte. Daraus schliesst nun Mikulicz, dass die mechanische Wirkung des Spray für die Wunden nur nachtheilig sein kann, da dadurch noch mehr Infectionskeime aus der Luft heruntergerissen werden, als wenn kein Spray angewendet wäre. Und er scheint auf den ersten Blick Recht zu haben, seine Experimente beweisend zu sein. Ueberlegen wir uns ’aber die Sache näher und vergegenwärtigen wir uns, unter welchen Bedingungen der Spray gewöhnlich, d. h. bei der Operation wirkt und unter welchen Bedingungen ihn Mikulicz wirken liess, so werden wir bald uns überzeugen, dass seine Experimente keine Beweiskraft haben können, weil sie falsch angelegt wurden. Sie würden wirklich zutreffend sein, wenn während unserer Operationen irgendwo in der Ecke des Operationszimmers ein Kobold sässe, der „continuirlich" frische Infectionskeime in die Luft streute; dies trifft aber doch in Wirklichkeit glicklicherweise nicht zu. Im Gegentheil befindet sich im Operationszimmer nur eine bestimmte Menge von Infectionskeimen. Für gewöhnlich ist der Spray, namentlich der Dampfspray, schon einige Zeit vor dem ersten Schnitt in Thätigkeit. In den ersten 5 Minuten fallen in dem besprayten Operationsterrain wirklich mehr Infectionskeime herab, als dies ohne Spray geschehen würde; es sind dann im Operationszimmer - und namentlich in nächster Operationsnähe - so viel weniger Infectionskeime, als mehr herabgefallen sind, dies wiederholt sich in den zweiten 5 Minuten; in den dritten und folgenden 5 Minuten ist aber dadurch die Luft in der nächsten Umgebung der Wunde so gereinigt, dass trotz eines langsamen Zuströmens der Infeetionskeime aus weiteren Regionen des Operationszimmers - wenn wir auch von dem durch den Spray erzeugten abwehrenden Luftstrom absehen - dennoch viel weniger Infectionskeime auf die Wunde herabfallen, als wenn der Spray nicht gewirkt hätte, ja, ibre Menge fast gleich Null wird.

Wäre nur Mikulicz bei dem Beispiel, das er am Eingang bei den Betrachtungen der mechanischen Wirkung des Spray gewählt, geblieben und hätte es richtig aufgefasst, so würde auch er ohne Weiteres die Richtigkeit obiger Deductionen eingeseben haben. Er bemerkt nämlich, dass der atmosphärische Regen die Luft durch 
Herunterreissen der suspendirten Staubtheilchen reinigt und fuigt hinzu, dass es jede Hausfrau auf dem Lande weiss, "dass das erste Regenwasser sehr bald zu faulen anfängt", und schliesst daraus, dass wenn der Spray mit Recht mit dem Regen verglichen wird, seine mechanische Wirkung nur zu Ungunsten desselben ausfällt, vergisst aber, dass nur das „erste“ Regenwasser sebr bald zu faulen anfängt, während das nächste desto länger davon frei bleibt.

Um uns von der Richtigkeit unserer obigen Deductionen zu überzeugen, haben auch wir mit Kohlenstaub Versuche angestellt, dieselben aber entsprechend den bei der Operation gegebenen Bedingungen eingerichtet.

Erste Versuchsreihe.

Wir haben in einem Zimmer eine bestimmte Kohlenstaubmenge ausgestäubt. Am anderen Ende des Zimmers wurden nach einander fünf flache Gefässe je 3 Minuten in etwa $11_{12}$ Meter Entfernung von Dampfspray unter denselben aufgestellt. $\mathrm{Zu}$ gleicher Zeit wurden hinter den Spray auf' gleiche Zeit je ein Controlteller aufgestellt und dann verglichen, wie sich die Kohlenstaubmengen auf dem der Wirkung des Dampfspray ausgesetzten Teller zu denen des Controltellers verbielten :"

1. Nach den ersten 3 Minuten war auf dem Sprayteller entschieden mehr Kohlenstaub, wie auf dem Controlteller. (Stimmt mit den Mikulicz'schen Versuchen überein.)

2. Der Unterschied auf den die nächsten 3 Minuten aufgestellten Tellern an Kohlenstaubgehalt war häufig so gering, dass man nicht genau unterscheiden konnte, wo mehr war; in der Mehrzahl der Versuchsfälle schien etwas mehr auf dem Sprayteller zu sein. Ueberhaupt aber war auf jedem Teller weniger Kohlenstaub wie auf den ersten.

3. In dem die dritten 3 Minuten dem Spray ausgesetzten Teller war ganz entschieden weniger Kohlenstaub, wie im Controlteller, in beiden Tellern aber noch weniger Kohlenstaub als bei 2 .

4. Auch bei den vierten 3 Minuten war noch wahrnehmbar, dass auf dem Sprayteller weniger Kohlenstaub, als auf dem Controlteller sich befand, - auf beiden aber überhaupt viel weniger, wie bei lden vorigen.

5. Auf beiden Tellern ist schon so wenig Kohlenstaub, dass ein bestimmter Unterschied nicht mehr wahrnehmbar war.

$$
\text { Zweite Versuchsreibe }
$$

wurde in ähnlicher Weise mit dem Handspray angestellt. Die Ergebnisse waren hier dieselben, nur war in einigen Fällen der Unter- 
schied erst beim vierten Tellerpaar am deutlichsten, was iubrigens auch manchmal bei Anwendung des Dampfspray variirte.

Wenn es erlaubt ist, diese grob mechanischen Verhältnisse auf die feinen Infectionskeime zu uibertragen, so beweisen sie zur Evidenz die Richtigkeit unseres obigen aprioristischen Raisonnements. Eine gewisse Beweiskraft wird man ihnen nicht absprechen dürfen, da sie genau den im Operationszimmer gegebenen Verbältnissen Rechnung tragen.

Wir sind also durch theoretische Erwägungen und Experimente gerade zu der entgegengesetzten Ansicht, wie Mikulicz gekommen und dürfen, glaube ich, mit Reeht behaupten, dass die mechanische Wirkung des Spruy der Wunde sehr wohl nülzt, namentlich wennwie gewöhnlich - dor Spray cinige Zeil vor dem ersten Sclmill oder dem Fortnelmen der letzten Schicht des Verbandes in Thätiglicit gesetzl ist.

Wir wïrden daraus zugleich den Rath entnehmen, dies immer zu thun. Diese Wirkungsweise des Spray ist, wie schon erwähnt, auch durch die häufigste Irrigation nicht zu ersetzen, also ist dadurch allein schon vom theoretischen Standpunkt aus bewiesen, dass der Spray nicht vollständig durch die Irrigation zu ersetzen ist.

Die zweite Frage ist nach der chemischen Wirkung des Spray.

Da unterscheidet Mikulicz (S. 73T) ganz richtig eine doppelte antiseptische Wirkung:

1. Werden alle Luftkeime dureh den Contact mit dem Antisepticum in ihrer Lebensfähigkeit an und für sich herabgesetzt noch ehe sie zur Wunde herabfallen; passiren also gleichsam ein antiseptisches Bad schon in der Luft.

2. Die Wunde wird mit einer gewissen Menge des Antisepticums imprägnirt und dadurch zu einem ungünstigen Boden für die Entwicklung der Infectionskeime umgestaltet.

Nach Mikulicz's Ansicht erfillt genau denselben Dienst, wie der Spray, die einfache Irrigation.

Er glaubt nämlich nicht, dass es von Belang sei, dass die Luftkeime schon vor dem A uffallen anf die Wunde beim Anwenden des Spray von dem Antisepticum getroffen werden, weil er der Ansicht ist, dass die übliche Concentration desselben nicht ausreicht, einfach durch Contact die Entwicklungsfähigkeit der Luftkeime zu zerstören. Den Beweis dafür blieb er schuldig und dürfte es kaum glaublich erscheinen, dass das Bespülen der Luftkeime mit $2-3$ proc. Carbollösung vollständig ohne hemmende Wirkung auf ibre Entwicklungsfähigkeit sein sollte, wenn andererseits oft schon "ganz ge- 
ringe" Mengen eines Antisepticums binreichen sollen, um den Nährboden, auf welchen die Pilze auffallen, für eine längere Zeit hindurch für die Keimung ungünstig zu gestalten; was Mikulicz durch eine Reihe von Experimenten nachgewiesen haben will. Nach diesen Versuchen fand Mikulicz, dass die halbständige Einwirkung des Carbolspray bei alkalisirtem Urin die Entwicklung der Luftkeime verzögerte, und in Pasteur-Bergmann'scher Lösung in der Mehrzahl der Fälle vollständig verhinderte. Dieses soll die in die Näbrflüssigkeit niedergefallene Carbolsäure bewirkt haben, deren Quantitäten 0,02 - 0,03 Proc. betrug. Mit Recht - glauben wir - fügt Maas dem Referat darïber (1. c.) hinzu, dass ein Zusatz von 0,02-0,03 proc. Carbolsäure nicht im Stande ist, die Entwicklung von Bacterien in einer Nährflüssigkeit zu verbinden.

Nach obigen Auseinandersetzungen wird man uns zugeben miissen, dass die chemische Wirkung des Antisepticums auf die Luftkeime vor ihrem Herabfallen auf die Wunde nicht vollständig ausser Acht zu lassen ist; und mag sie auch nicht besonders gross sein, so ist sie jedenfalls durch die nachträgliche Irrigation nicht zu erreichen.

Wir sind mit Mikulicz u. A. uiberzeugt, dass die Luftkeime mindestens mebrerer Stunden bedürfen zum Beginn ihrer Entwicklung und deshalb noch nachträglich durch die nachfolgende Irrigation erreicht werden könnten, wenn die Irrigationsflüssigkeit alle Winkel und Buchten der Wunde erreichen und aus ibnen die Bacterien immer sicher herausspülen würde; wenn es absolut nicht vorkommen könnte, dass eine Anzahl derselben entweder in einem Winkel unerreicht bleiben oder durch die späteren Operationsmanipulationen in die Tiefe der Gewebe hineingepresst werden könnte. Dieses wird in der Praxis vielleicht sehr selten zutreffen; wozu sich aber eines Sicherheitsmittels, wenn aucb für die seltensten Fälle, berauben, zumal da es für mich jedenfalls noch zweifelhaft erscheint - wie schon oben erwähnt - was unbequemer für den Operateur ist: der Spray oder das Irrigiren - namentlich das häufigere während der Operation. - Vom theoretischen Standpunkte aus glaube ich bewiesen zu haben, dass die Irrigation auch in obiger Beziehung nicht im Stande ist, vollständig den Spray zu ersetzen.

Dazu kommt noch, dass bei einzelnen Wunden wie bei denen des Peritoneal- und Pleurasackes die nachträgliche Irrigation unstatthaft ist. Freilich behauptet Mikulicz, dass "das normale Peritoneum selbst unter gewöhnlichen Verhältnissen von der Luft aus iberhaupt nicht inficirt werden kann"; den Beweis dafur will er bei anderer 
Gelegenheit geben. Nun muss man aber zugeben, dass wir nicht selten gezwungen sind, die Peritonealhöhle zu öffnen, auch wenn das Peritoneum nicht "normal " ist und häufig auch unter "nicht gewöbnlichen "Verhältnissen. Ueberdies ist es nicht selten passirt, dass Eiterung in der Peritonealhöhle aufgetreten ist, nachdem man Exsudate derselben punctirt hat, und zwar punctirt hat mit Troikaren, die längere Zeit in 5 proc. Carbollösung lagen und nachdem man die Bauchhaut mit derselben Lösung abgewaschen hat, also eigentlich doch am wahrscheinlichsten blos angeregt durch die Infectionskeime aus der eingedrungenen Luft. In dem nachfolgenden casuistischen Beitrag werde ich zeigen, dass ich aus besonderen Grïnden gerade bei Laparotomien während der Operation den Spray nicht anwende, aber da suche ich mich durch andere besondere Vorsichtsmaassregeln - ebenso wie viele andere Operateure - vor der Infection $\mathrm{zu}$ schïtzen.

Noch schwächer ist der Beweis für die Entbehrlichkeit des Spray bei Pleurawunden ausgefallen: Ein Fall von KolaczekFischer wird angeführt und auf andere hingewiesen. Aber gerade bei gröberer Punction pleuritischer seröser Exsudate ist bei Luftzutritt erfahrungsgemäss häufig Vereiterung eingetreten, so dass man sich die grösste Mühe gegeben hat, Instrumente zu erfinden, welche den Luftzutritt sicher vermeiden liessen, weil man sich eben iberzeugt hat, dass hier die blosse Desinfection der Instrumente nicht ausreicht.

Die theoretischen Deductionen, die dem erwähnten Beispiele folgen, haben keine Beweiskraft, weil sie etwas als bewiesen annehmen und zur Argumentation benutzen, was zu beweisen ist:

Mikulicz geht davon aus, dass der Spray auf die Infectionskeime in der Luft keinen Einfluss hat und blos dadurch wirkt, dass dessen Antisepticum auf den Näbrboden sterilisirend einwirkt. Da aber durch eine kleine Oeffnung nur wenig des Antisepticums aus dem Sprühregen in die Pleurahöhle gelangen kann, so wird dieses auf der weiten serösen Fläche sehr rasch durch Diffusion und Resorption bis zur Unwirksamkeit verdiunt (wohl unter 0,02-0,03 Proc.). Gerade hier dürfte es einleuchtend sein, dass ein längere Zeit vor Eröffnung der Pleurahöhle auf die betreffende Stelle gerichteter Spray, der auch während der Operation thätig bleibt, sehr nützlich sein muss, seine oben besprochene Wirkungsweise segensreich entfaltet.

Ich glaube durch die vorangegangenen theoretiscben Auseinandersetzungen und die angeführten Experimente hinlänglich bewiesen zu haben: 
1) dass der mechanische Effect des Spray der. Wunde nicht schadet, sondern nutzt; 2) dass die nachfolyende Imigation wenigstens nicht in allen Füllen den Spray ersetzen liann, da sie nicht alle wirksamen Eigenschaften desselben vertritt und zweitens nicht überall angewendet werden liann.

Zum Schluss will ich nur noch mit kurzen Worten darauf aufmerksam machen, dass man die Wirkung des Spray nicht ausschliesslich auf den Schutz vor der Luftinfection beschränken darf und er dadurch noch mehr an Wichtigkeit gewinnt. Gerade bei den gewöhnlichen täglichen Operationen kommen wir so häufig während der Operation mit unseren Händen in Berülhrung mit Gegenständen, die wir nichts weniger als rein im aseptischen Sinne bezeichnen können: so mit den Kleidern, dem Operationstisch, Haaren u. s. w., die Instrumente, wie Pincetten legen wir nicht immer in das bestimmte Gefäss sondern nicht selten nebenbei auf den 'Tisch. Es könnte sich da nicht selten ereignen, dass bei dem nachherigen Manipuliren ein Infectionsstoff so tief und fest in die Wunde eingepflanzt würde, dass er nachträglich gar nicht abgeschwemmt werden könnte, während der fortwährend auf unsere Hand und Instrumente auffallende Spray die Infectionskeime, wenn nicht immer vor ihrem Gelangen zur Wunde abspült, so doch mit dem Antisepticum abwäscht, sie sterilisirt.

Der Zweck dieser Arbeit soll wenigstens der sein, dass man nicht allzu rasch mit dem Spray abrechnet. Die praktischen Erfolge Trendelenburg's und v. Bruns' sind sehr schön, aber man darf nicht - wie schon Eingangs erwähnt - vergessen, dass sie in Kliniken erreicht sind, in denen das antiseptische Verfabren seit Jahren streng geübt worden ist, wo seit langer Zeit accidentelle Wundkrankheiten gar nicht oder höchst selten vorgekommen, wo alle Räume mit Carbolsäure gleichsam iiberfiillt, wo endlich Operateur und Assistenten in bester Weise das iubrige antiseptische System nach Fortlassung des Spray iiben konnten und wollten. Da rächt sich so Manches nicht. Aber man denke sich ein überfïlltes Kriegslazareth, wo häufig Wunden hinkommen, die nicht aseptisch sind, wo viel des Infectionsstoffes angehäuft ist; wo der Operateur nicht immer bei der Masse von Arbeit auf die minutiöseste Reinlichkeit der Instrumente u. s. w. achten kann, wo Assistenten und Wärter nicht immer vollständig bekannt sind mit dem aseptischen Verfahren und häufig - wenn sie es auch sind - nicht immer Zeit haben all' die nöthigen Cautelen anzuwenden - ich meine in solchen Verhältnissen wird nicht selten der Spray noch in der letzten 
Minute die Correction übernehmen. - Und doch haben die nevesten Arbeiten auch den Zweck zu beweisen, dass gerade für den Krieg der Spray sehr lästig und nicht nöthig ist.

Deshalb möchte ich am Schluss meiner Arbeit ausrufen :

"Nur nicht allzu ubereilt fort mit dem Spray!"

\section{Zehn Fälle ron Laparotomic.}

Der nachfolgende easuistische Beitrag, dessen einzelne Fälle an und für sich einiges Interesse beanspruchen, soll zugleich zeigen, dass ich nicht einseitig zu Gunsten des Spray eingenommen bin und sollen dadurch den Werth der vorangegangenen Erörterungen erhöhen.

Wir haben bei all den in nicht gan $1_{1 / 2}^{1}$ Jahren unternommenen Laparotomien, deren Zahl 10 beträgt, den Spray während der Operation weggelassen, weil wir zu der Ansicht Derjenigen neigen, welche die Abkïhlung der Baucheingeweide durch den Spray und die Reizung derselben durch die zerstäubte Carbolsänre fürchten.

Wir haben in keinem einzigen Falle bei unseren Operirten bestimmt Peritonitis als Todesursache beobachtet. Folgende Arten von Laparotomien sind angeführt:

1-3. Drei Ovariotomien: zwei mit sehr zahlreichen Adbäsionen neben ungewölınlich grossen Tumoren.

4 und 5. Zwei totale Uterusexstirpationen, von denen eine letal endete.

6. Eine Castration.

๘. Eine Exstirpation des carcinomatösen Pylorus mit letalem Ausgang.

8 und 9. Zwei Probeincisionen bei Abdominaltumoren.

10. Eine Fistula stercoralis geheilt durch Enterorrhaphie.

Von diesen Laparotomien sind also zwei gestorben, aber beide an Schwäche und nicht in Folge von Infection. Trotzdem bin ich weit entfernt, daraus den Schluss ziehen zu wollen: weil von 10 ohne Spray ausgefilihrten Laparotomien alle aseptisch verlaufen, so ist der Spray bei dem antiseptischen Verfahren unnöthig. - Im Gegentheil nur dadurch, dass ich mir wohl bewusst war von der Wichtigkeit desselben, glaube ich die guten Resultate in Bezug anf Asepsis erreicht zu haben; denn in Folge dessen führte ich beim Fortlassen des einen Schutzmittels desto peinlicher die anderen aus und suchte 